\title{
BIBLIOTECA MATEMATICA
}

\author{
PUBBLICAZIONI NON PERIODTCHE \\ PERVENUTE IN DONO AL CIRCOLO. \\ $\mathrm{XIX}^{\circ}$ Elenco: (agosto r gor - dicembre I 902). \\ [Vedi gli Elenchi precedenti: t. XV, pp. 30-34 e retro].
}

Alasia, C. (Città di Castello). Poligeometrognomia generale e la geometria non-euclidea del Chrystal. Rivista Sc. e Lett. (Quo Vadis?) di Trapani. Anno I e II.

- Une méthode élémentaire de recherche des maxima et minima et ses applications. Gazeta matematica, I902.

- Alcune osservazioni sui pendoli e sui cronometri. Rassegna Technica. Anno II, 1902.

- H. Faye. Rivista di Fis. Mat. e Sc. di Pavia. Anno III.

- Saggio terminologico-bibliografico sulla recente geometria del triangolo. Bergamo, 1902.

Amato, V. (Catania). [Vedi t. XV, p. 30]. Sull'integrazione di talune equazioni a derivate parziali di $2^{\circ}$ ordine. Atti Acc. Gioenia di Sc. Nat. in Catania, vol. $\mathrm{XVI}_{4}$.

Amodeo, F. (Napoli). [Vedi t. XV, pag. 30]. Stato delle matematiche a Napoli dal 1650 al 1732. Napoli, 1902.

- Coup d'œil sur les courbes algébriques au point de vue de la gonalité. Deuxième Congrès Int. des Math., Paris, I9oo.

- Rappresentazione stereoscopica delle figure dello spazio nel piano. Le Mat. pure ed appl., Anno II, 1902.

- Le riforme universitarie di Carlo III e Ferdinando IV di Borbone. Atti Acc. Pont., vol. XXXII.

Andoyer, H. (Paris). [Vedi t. XIV, pag. 1]. Théorie de la Lune. Paris, 1902.

Rend. Circ. Malem. Palerma, t. XVI, parte $2^{a}$ (1902). - Stampato il 31 dicembre 1902. 
Atti del congresso di insegnanti di scuole medie tenuto in Palermo il 30 e $3^{I}$ Maggio Ig02. Palermo, 1902.

Balbi, V. (Torino). Osservazioni meteorologiche fatte nell'anno rgor all'Osservatorio della R. Università di Torino. R. Acc. Sc. Torino, rgor-rgo2.

Barbarin, P. (Paris). La géométrie non euclidienne. Paris, Igoz.

Bardelli, G. (Milano). [ $V$ edi t. XIV, pag. 2]. Su un teorema statico di Leibniz. R. Ist. Lomb. Sc. e Lett., vol. $\mathrm{XXXV}_{2}$, 1.902.

Beltrami, E. [Vedi t. VII, pag. I]. Opere matematiche, t. I. Ulrico Hoepli. Milano, 1902.

Bianchi, L. (Pisa). [Vedi t. XIII, pag. 2]. Lezioni di geometria differenziale. $2^{2}$ ed. riveduta considerevolmente aum»ntata, vol. I, Pisa, 1902.

Boccardi, G. (Catania). [Vedi t. XV, pag. 30]. Catalogo di stelle fondamentali fra $+46^{\circ} \mathrm{e}+55^{\circ}$. Equinozio del 1900,0. R. Osservatorio di Catania. Catania, I90 I.

Borel, E. (Paris). [Vedi t. XV, pag. 30]. Leçons sur les séries à termes positifs. Paris, 1902.

Brioschi, F. [Vedi t. XV, pag 30]. Opere matematiche, t. II. Ulrico Hoepli. Milano, 1902.

Burali-Forti, C. (Torino). [Vedi $\downarrow . \mathrm{XV}$, pag. $3 \mathrm{I}$ ]. Sopra alcuni punti singolari delle curve piane e gobbe. $R$. Acc. delle Sc. di Torino, rgoo-rgor.

- Le formule di Frenet per le superficie. Ibid., Igor-Igo2.

- Logique et histoire des sciences. Paris, Armand-Colin.

Burgatti, P. (Cento). [Vedi t. XIV, pag. 3]. Sopra un teorema di Levi-Civita riguardante la determinazione di soluzioni particolari di un sistema Hamiltoniano. Rend. Acc. Lincei, vol. $\mathrm{XI}_{\mathrm{s}}, \mathrm{I}^{\circ}$ sem. 1902.

Capelli, A. (Napoli). [Vedi t. XV, pag. 31]. Lezioni sulla teoria delle forme algebriche. Napoli, 1902.

- Il concetto di valore e l'introduzione nell'aritmetica dei numeri negativi e frazionarii. Giorn. di Battaglini, vol. XXXIX, Igor.

- Sulla riduttibilità della funzione $x^{n}-A$ in un campo qualunque di razionalitat. Math. Annalen, vol. LIX, Igor.

- Sulla continuità delle funzioni di più variabili reali. Rend. della $R$. Acc. delle Sc. Fis. e Mat. di Napoli, 1902.

- Le iper-aritmetiche e l'indirizzo combinatorio dell'aritmetica ordinaria. Deuxième Congrès int. des math., Paris, rgoo.

- Istituzioni di analisi algebrica, $3^{\mathbf{a}}$ ed. con aggiunte delle Lezioni di algebra complementare. Napoli, I902.

Carvallo, E. (Paris). L'électricité déduite de l'expérience et ramenée au principe des travaux virtuels. Paris, I902.

Cipolla, M. (Palermo). La determinazione assintotica dell' $n^{\text {mo }}$ numero primo. Rend. R. Acc. delle Sc. Fis. e Mat. di Napoli, 1902.

Combebiac, G. (Paris). Thèses présentèes à la Faculté des Sciences de Paris. $\mathrm{I}^{\mathrm{re}}$ : Calcul des triquaternions. $2^{\mathrm{e}}$ : Propositions données par la Faculté. Paris, igo2. 
Coulon, J. (Paris). Thèses présentées à la Faculté des Sc. de Paris pour obtenir le gradede docteur ès Sc. math. $\mathrm{I}^{\mathrm{re}}$ Thèse: Sur l'intégration des équations aux dèrivies purtielles du $2^{\mathrm{e}}$ ordre par la méthode des caractéristiques. $2^{\mathrm{e}}$ Thèse: Propositions données par la Faculté. Paris, 1902.

Dall'Acqua A. (Venezia). Sulla teoria delle congruenze di curve in una varietà qualunque a tre dimensioni. Annali di Matem., vol. $\mathrm{VI}_{3}$, rgor.

Duporcq, E. (Paris). [Vedi t. XIV, pag. 4]. Compte rendu du deuxième Congrès international des Mathematiciens, tenu a Paris du 6 au I 2 aout Igoo. Paris, 1902.

Fano, G. (Messina). [Vedi t. XV, pag. $3 \mathrm{I}$ ]. Le congruenze di rette del $3^{\circ}$ ordine composte di tangenti principali di una superficie. R. Acc. Sc. Torino, t. XXXVII, 1901-1902.

Forsyth, A. R. (Cambridge). [ $V e d i$ t. XIII, pag. 4]. Theory of Differential Equations. Part. III, ordinary linear equations. Vol. IV. Cambridge, 1902.

Gebbia, M. (Palermo). [Vedi t. VII, pag. 4]. Le deformazioni tipiche dei corpi solidi elastici. An. di Mat. pura ed app., t. $\mathrm{VII}_{3}$, 1902 .

Giudice, F. (Genova). [Vedi t. XIV, pag. 4]. Teoremi relativi alla convergenza e divergenza delle serie numeriche. Le mat. pure ed app., t. II, Igo2.

Godefroy, M. (Marseille). [Vedi t. XV, pag. 32] Théorie élémentaire des séries. Limite - Séries à termes constants - Séries à termes variables - Fonction exponentielle - Fonctions circulaires - Fonction gamma. Paris, 1903.

Goursat, íd. (Paris). [Vedi t. XIII, pag. 5]. Cours d'analyse mathématique. Tome I: Derivées et différentielles. Intégrales définies. - Développements en sérias. Applications géométriques. Paris, rgoz.

Guccia, G. B. (Palermo). [Vedi t. X, pag. 8]. Sulle curve algebriche piane. Rend. Circ. Matem., t. XVI.

- Sulle superficie algebriche. Ibid., t. XVI.

Hedrick, E. R. (Michigan). Ueber den analytischen Character der Lösungen von Differentialgleichungen. Göttingen, rgor.

Jamshedji, Ed. (Ahmedabad). [Vedi t. XIV, pag. 4]. Reciprocally related Figures and the Property of Equianharmonicity. Ahmedabad, rgor.

Laurent, H. (Paris). [Vedi t. XIV, pag. 4]. Sur les principes fondamentaux de la théorie des nombres et de la géométrie. Coll. SG. $\mathrm{n}^{\circ}$ 20. Paris, I902.

Lebon, E. (Paris). [Vedi t. XV, pag. 32]. Notice sur les travaux mathematiques de M. ERnest Lebon. Paris, 1900.

Lemoine, E. (Paris). [Vedi t. XV, pag. 32]. Géométrographie ou art des constructions géométriques. Paris, 1902.

Loria G. (Genova). [Vedi t. XV, pag. 32]. Eugenio Beltrami e le sue opere matematiche. Bibliotheca mathemalica, Bd. $\mathrm{II}_{3}$.

- Le curve panalgebriche. Mém. de la Soc. R. des Sc. de Bohême. Prague, 19or.

- Le scienze esatte dell'antica Grecia. Libro $V$ (ultimo): L'aritmetica dei Greci. Modena, 1902.

- Donne Matematiche. Mem. R. Acc. Virg., 1901. 
- Pseudo versiera e quadratrice geometrica. Bibl. Mat., Bd. III.

- Le quadrisecanti di una quaterna di rette. Periodico di Mat., t. XVII.

- L'œuvre mathématique d'Ernest de Jonquiéres Bibl. Math., Bd. III 3 , 1902.

- Elenco delle pubblicazioni matematiche di Ernesto de Joneuières. Boll. di Bibl. e storia delle Sc. Mat., 1902.

Macé de Lépinay J. (Marseille). Franges d'interférence. Paris, 1902.

Marangoni G. B. (Bassano Veneto). [Vedi t. XIV, pag 5]. Note critiche su alcune recenti pubblicazioni scolastiche. (Per il II Congresso di "Mathesis »). Bassano, rgor.

Marletta, G. (Catania). Sulla varietà delle rette contenute in una o più forme algebriche. Atti Acc. Sc. Catania, vol. $\mathrm{XVI}_{4}$.

Martin E. N. (Elizabeth). In the imprinitive substitution groups of degree Fifteen and the primitive substitution groups of degree eighteen. Baltimore, rgor.

Martinetti, V. (Messina). [Vedi t. XIII, pag. 8]. Un gruppo di Cfr. $\left(9_{4}, 9_{4}\right)$ di punti e piani. R. Acc. Peloritana. Anno XV, Igor.

- Alcune considerazioni sulla configurazione di Kummer. Rend. Circ. Mat., t. XVI.

Matiegka, H. Bericht über die Untersuchung der Gebeine Tycно BraHz's. Sitzung. k. böhm. Gesellsch. der Wissensch., Igor.

Mayer, A. (Leipzig). [Vedi t. XIV, pag. 5]. Simmetrische Lösung der Aufgabe, die Rotation eines starren Körpers, dessen Winkelgeschwindigkeiten bereits gefunden wurden, vollstāndig zu bestimmen. Ber. der math.-phys. Classe der Kön. Säch. Ges, der Wiss. zu Leipzig. 1902.

- Ueber den Zusammenstoss zweier Körper unter Berücksichtigung der gleitenden Reibung. Ibid.

- Nachtrag zu dem Aufsatze: "Ueber den Zusammenstoss zweier Körper unter Berūcksichtigung der gleitenden Reibung». Ibid.

Mignosi, G. (Palermo). Un problema sulla partizione dei numeri. Per. di Mat., volume XVIII, Ig02.

Molk, J. Tannery, J. (Nancy, Paris). [Vedi t. XIII, pag. Io]. Eléments de la théorie des fonctions elliptiques. Calcul Intégral (II partie). Applications, t. IV. Paris, 1902.

Néculcéa, E. (Paris). Le phénomèn de Kerr. Paris, Igo2.

Orlando, L (Messina). Note di Matematica. Messina, I902.

- Sulla velocità minima nella trajettoria d'un grave. Atti della $R$. Acc. Peloritana, anno XVIII, Igo2.

Paternó, F. P. (Palermo). [Vedi t. XIII, pag. 8]. Saggio di una teoria sull'approssimazione naturale o variabile delle radici quadrate. Period. di Matem., vol. XVII, Igor.

- Un teorema sulle potenze dei numeri interi. Suppl. al period. di Mat., t. V.

Pennacchietti, G. (Arcevia). [Vedi t. XV, pag. 32]. Sugli integrali comuni a più problemi del moto di un punto materiale sopra una superficie. Atti Acc. Catania, vol. $\mathrm{XV}_{4}, 1902$.

- Sulle equazioni differenziali del moto di un corpo solido intorno ad un punto fisso. Ibid., vol. $\mathrm{XVI}_{4}$, I902. 
Perrin, R. (Paris). [Vedi t. V, pag. 7]. Sur le covariant résolvant de la forme binaire du cinquième ordre. $2^{\text {e }}$ Congrès Int. des Math., Paris, I900.

- Sur une méthode nouvelle pour la séparation et le calcul approximatif des racines réelles des équations numériques. Comp. rendus de l'Ass. Fran. pour l'Av. des Sc., Congrès d'Ajaccio, I9or.

Picard, É. (Paris). [Vedi t. XV, pag. 33]. Quelques réflexions sur la mécanique, suivies d'une première leçon de dynamique. Paris, 1902.

Pincherle, S. (Bologna). [Vedi t. VIII, pag. 8]. Le operazioni distributive e le loro applicazioni all'analisi. Bologna, rgor.

Raoult, F. M. (Grenoble). Cryoscopie. Paris, Igor.

Reina, V. (Roma). [Vedi t. XIII, pag. 9]. Determinazione di latitudine e di azimut eseguite nel 1898 nei punti M. Mario e M. Cavo-Fiumicino. $R$. Comm. Geod. Ital, 1899 .

- Determinazione astronomica di latitudine e di azimut eseguita a M. Pisarelio nel I899. Rend. Acc. Lincei, vol. IX, I900.

- Gli strunenti di ottica e di meccanica di precisione all'esposizione universale del rgoo. Riv. di Top. e Catasto, vol. XIII, Torino, Igoo.

- Determinazione astronomiche di latitudine e di azimut eseguite a Roma (S. Pietro in Vincoli) a M. Cimrno ed a M. Pegria negli anni igoo e Igor. Rend. Acc. Lincei, vol. $\mathrm{XI}_{5} \mathrm{I}^{\circ}$ sem. 1902.

Riccò, A. (Catania). [Vedi t. XV, pag. 33]. Nova (3. 1901) Persei. Osservazioni astrofisiche fatte nel R. Osservatorio di Catania. Mem. Soc. Spettrosc., vol. XXX, 1901.

- Deformazione del sole all'orizzonte. Ibid.

Robin, G. CEuvres scientifiques, réunies et publiées par L. Raffy. Physique mathématique. Paris, 1899.

- Id. Thermodynamique générale. Paris, I90I.

Servais, C. (Gand). Sur les faisceaux de coniques. Le Matem. pure ed app., anno I, Igor.

Silberstein, $\cdot$ L. Symbolische Integrale der elektromagnetischen Gleichungen, aus dem Anfangszustand des Feldes abgeleitet, nebst Andeutungen zu einer allgemeinen Theorie physikalischer Operatoren. Annalen der Physik, Folge IV, Bd. 6, rgor.

Somigliana, C. (Pavia). [Vedi t. XV, pag. 33]. Sul principio delle immagini di Lord Kelvin e le equazioni dell'elasticita. Rend. Acc. Lincei, t. $\mathrm{XI}_{5}, \mathrm{I}^{\circ}$ sem., I9O2.

- Sul potenziale elastico. Ann. di mat., t. $\mathrm{VII}_{3}$, 1902.

Strazzeri, V. (Sassari). Le eliche cilindriche. Sassari, Igor.

Studnička, F. J. (Praga). [Vedi t. XIV, pag. 7]. Bericht über die astrologischen Studien des Reformators der Beobachtenden Astronomie. Tycho Brahe. Prag, rgor.

Torelli, G. (Palermo). [Vedi t. XI, pag. 90]. Sulla totalità dei numeri primi fino ad un limite assegnato. Atti Acc. Napoli, vol. $\mathrm{XI}_{2}$, $190 \mathrm{I}$.

Tychonis Brahe Dani. De nova stella. Edidit Regia Societas Scientiarum Danica. Hauniae, MDCCCCI. 
Vendeuren, P. (Bruxelles). Étude géométrique des lignes et des surfaces en un point ordinaire. Représentation géométrique des derivées. Bruxelles, 1902.

Verde, F. (Spezia). [Vedi t. XI, pag. 9I]. Osservazioni astronomiche a bordo con orizzonte artificiale. Rivista Scientifico-Industriale. Firenze.

Vivanti, G. (Messina). [Vedi t. XIV, pag. 7]. Programmi e riassunti di corsi universitari. Bollett. di Bibliografia, 19or.

- Lezioni sulla teoria degl'integrali Abeliani, tenute nella $R$. Università di Messina e raccolte dagli studenti. Corso dell'anno r900-1901. (Litogr.). Reggio Calabria, rgor.

- Teoria delle funzioni analitiche. Milano, rgor.

- Il concetto d'infinitesimo e la sua applicazione alla matenatica. Giornale di Matematica, t. XXXVIII, XXXIX.

- Sopra le rotazioni della sfera su sè stessa. Le Mat. pure ed appl., t. II.

- Lezioni sulla teoria della risoluzione delle equazioni di $5^{\circ}$ grado tenute nella R. Università di Messina e raccolte dagli studenti. Messina, IgoI-02.

Vries, Jan de (Amsterdam). [Vedi t. XV, pag. 34]. La configuration formée par les vingt-sept droites d'une surface cubique. Arch. Nierl.

- Ueber die Simultaninvarianten zweier Kegelschnitte. Niew Archief voor Viskunde, $\mathrm{Bd}, \mathrm{V}_{2}$, rgor.

- The number of conics intersecting eight given right lines. Ak. van Wetensch., Amsterdam, rgor.

- A formula for the volume of the prismoid. Ibid., 1902.

Weyr, Ed. (Praga). [Vedi t. XIII, pag. II]. Pocět Differenciallny. Praze, rgoz.

Williot, V. (Paris). Études sur les nombres ptemiers. $I^{\text {re }}$ partie: $\mathrm{La}_{2}$ voie de RiEMaNN. Paris, 1903.

Zeuthen, H. G. (Copenhague). [Vedi t. XIII, pag. II]. Histoire des mathématiques dans l'antiquité et le moyen âge. Paris, 1902.

RODOLFO TAGIIARINI. 